\title{
Total laparoscopic pylorus-preserving pancreatoduodenectomy in an 89-year-old man: A case report and review of a single institute's experience in elderly patients
}

\author{
HONGYU LI and BING PENG \\ Department of Pancreatic Surgery, West China Hospital, Sichuan University, Chengdu, Sichuan 610041, P.R. China
}

Received December 31, 2014; Accepted February 1, 2016

DOI: $10.3892 / 01.2016 .4226$

\begin{abstract}
Total laparoscopic pylorus-preserving pancreatoduodenectomy (tLPPPD) has been demonstrated to be a safe and feasible surgery for pancreatic malignant diseases located in the head or uncinate process, with the advantages including minimal invasion, lower blood loss and a shorter hospital stay, compared with traditional open pancreatoduodenonectomy. Elderly patients theoretically have a lower capability to tolerate complex surgeries. The impairment of heart and pulmonary reserve function often leads to a high risk of post-operative cardiopulmonary complications. The present study reports a case of tLPPPD that was successfully performed in an 89-year-old man. No fatal complications developed. The post-operative pathological result revealed a diagnosis of pancreatic uncinate adenocarcinoma (T2N0M0, stage IB). After 1 year of follow-up, the patient remained alive without tumor recurrence or metastasis. The present study also discusses the associated literature and concludes that tLPPPD is a safe and feasible procedure in selective elderly patients. Use of this technique may expand the number of patients who can undergo surgery and provide benefits to these patients.
\end{abstract}

\section{Introduction}

Total laparoscopic pylorus-preserving pancreatoduodenectomy (tLPPPD) was first described by Gagner and Pomp in 1994 (1). Due to the complicated surgical procedure, tLPPPD is only performed in a few highly specialized hospitals. For pancreatic malignant diseases located in the head or uncinate process, tLPPPD has been demonstrated to be a safe and

Correspondence to: Professor Bing Peng, Department of Pancreatic Surgery, West China Hospital, Sichuan University, 37 Guoxue Street, Wuhou, Chengdu, Sichuan 610041, P.R. China E-mail: docpengbing@126.com

Abbreviations: tLPPPD, total laparoscopic pylorus-preserving pancreatoduodenectomy; POD, post-operative day

Key words: elderly patients, total laparoscopic pylorus-preserving pancreatoduodenectomy, complications feasible surgery (2). Previous studies showed a 1.6-1.8\% rate of mortality, perioperatively, in laparoscopic pancreaticoduodenectomy (LPD) $(1,2)$, which is similar to the $1 \%$ mortality rate associated with tLPPPD (3). Alternatives to tLPPPD include LPD, robot-assisted pancreaticoduodenectomy and hand-assisted pancreaticoduodenectomy. tLPPPD provides several advantages, including minimal invasion, lower blood loss and a shorter hospital stay, compared with traditional open pancreatoduodenonectomy (4). tLPPPD is most commonly performed in non-elderly patients (5). To date, few reports on the outcomes of tLPPPD performed in elderly patients, particularly those $>80$ years, have been published (6). Theoretically, age may be a significant prognostic factor for patients who undergo such complex surgery. An older age often indicates a worse tolerance for surgery, worse pre-operative conditions and a slower recovery. The present study reports a case of tLPPPD that was performed in an 89-year-old man and shares the experience of the West China Hospital (Chengdu, Sichuan, China) in treating such elderly patients.

\section{Case report}

An 89-year-old man was admitted to the Department of Hepatopancreatobiliary Surgery of West China Hospital in January 2014 with a 1-month history of jaundice. The physical examination was unremarkable, with the exception of skin and sclera jaundice. Laboratory results showed a total bilirubin (TB) level of $88.7 \mu \mathrm{mol} / 1$ (normal range, 5.0-28.0 $\mu \mathrm{mol} / \mathrm{l}$ ), a direct bilirubin (DBIL) level of $81.0 \mu \mathrm{mol} / 1$ (normal range, $<8.8 \mu \mathrm{mol} / \mathrm{l}$ ), a carbohydrate antigen $19-9$ level of $581.1 \mathrm{U} / \mathrm{ml}$ (normal range, $<22 \mathrm{U} / \mathrm{ml}$ ) and a normal coagulation test result. Upper abdominal computed tomography (Brilliance 64 Slice CT; Philips Medical Systems, Cleveland, OH, USA) scans showed dilation of the intrahepatic and common bile ducts. An arterial enhanced $3.5 \times 4.8-\mathrm{cm}$ mass was detected in the uncinate process, without evidence of superior mesenteric vessel invasion (Fig. 1). A malignant tumor of the uncinate process, with obstructive jaundice, was diagnosed pre-operatively. Due to a 10-year history of hypertension, an electrocardiogram, cardiac ultrasonography test and pulmonary function test were adapted to evaluate the patient. An atrial septal defect and atrial flutter were detected. Moderate cardiopulmonary impairment was subsequently diagnosed. tLPPPD is routinely performed for elderly patients $>70$ years in the West China Hospital. 
Table I. Previous studies that treated patients with total laparoscopic pylorus-preserving pancreatoduodenectomy.

\begin{tabular}{|c|c|c|c|c|c|c|c|c|}
\hline Authors & $\mathrm{n}$ & $\begin{array}{c}\text { Conversion, } \\
\%\end{array}$ & $\begin{array}{l}\text { Mortality, } \\
\mathrm{n}\end{array}$ & $\begin{array}{l}\text { Operation } \\
\text { time, min }\end{array}$ & $\begin{array}{c}\text { Pancreatic } \\
\text { fistula, \% }\end{array}$ & $\begin{array}{l}\text { Hospital } \\
\text { stay, days }\end{array}$ & $\begin{array}{l}\text { Negative-margin } \\
\text { resection, } \%\end{array}$ & Refs. \\
\hline Palanivelu et al, 2007 & 75 & 0 & 1 & 357 & 7 & 8 & 97 & $(10)$ \\
\hline Dulucq et al , 2006 & 13 & NA & 1 & 295 & 8 & 16 & 100 & (11) \\
\hline Zureikat et al, 2011 & 14 & 14.0 & 1 & 456 & 36 & 8 & 100 & (3) \\
\hline Kendrick and Cusati, 2010 & 65 & 4.6 & 1 & 368 & 18 & 7 & 89 & (2) \\
\hline Kim et al, 2013 & 105 & 4.7 & 1 & 474 & 6 & 11 & 100 & (5) \\
\hline
\end{tabular}

The definition of pancreatic fistula varies according to authors. NA, not available.

Table II. Elderly patients treated with total laparoscopic pylorus-preserving pancreatoduodenectomy at the West China Hospital, Sichuan University (Chengdu, China).

\begin{tabular}{lccccccc}
\hline Patient & $\begin{array}{c}\text { Age, } \\
\text { years }\end{array}$ & Conversion & Mortality & $\begin{array}{c}\text { Operation } \\
\text { time, min }\end{array}$ & $\begin{array}{c}\text { Pancreatic } \\
\text { fistula }\end{array}$ & $\begin{array}{c}\text { Hospital } \\
\text { stay, days }\end{array}$ & $\begin{array}{c}\text { Margin-negative } \\
\text { resection }\end{array}$ \\
\hline 1 & 77 & No & No & 488 & No & 12 & Yes \\
transfusion
\end{tabular}

Patient 5 is the patient reported in the present case study.

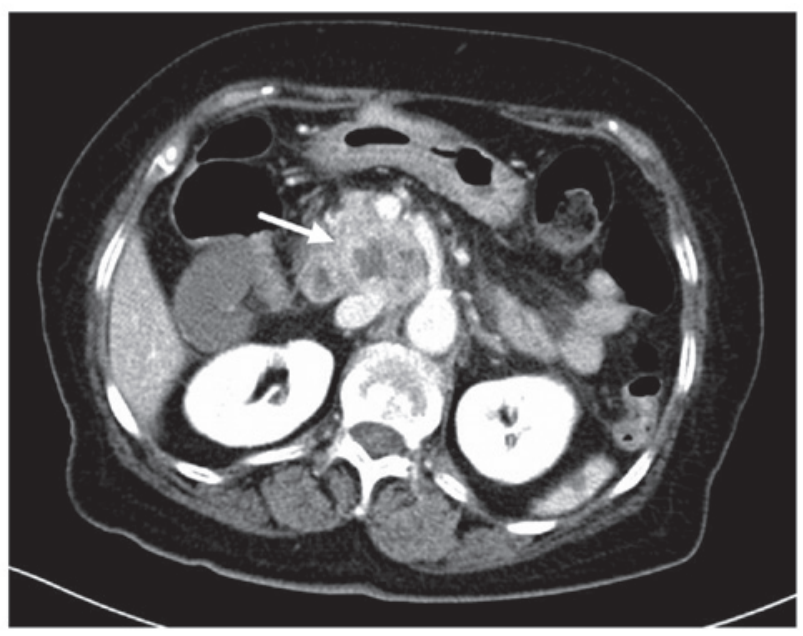

Figure 1. An arterial enhanced $3.5 \times 4.8-\mathrm{cm}$ mass located in the uncinate process of the pancreas (arrow).

Considering the high age and unsatisfactory pre-operative conditions of the present patient, tLPPPD was performed. For tLPPPD, the patients was placed in supine position with an anti-Trendelenberg $(10-30 \mathrm{~cm})$ position. Abdominal pressure using $\mathrm{CO}_{2}$ gas insufflation was maintained at $13-14 \mathrm{mmHg}$ and four trocars were placed under a direct vision scope. Then resection and reconstruction began For the resection, the gastrocolic omentum was dissected to allow entry into the lesser sac. The right gastroepiploic vessels were transected and the superior mesenteric portal vein was identified at the inferior border of the pancreas. The duodenum was mobilized and divided to the pylorus using an endoscopic linear stapler. A cholecystectomy was performed separately and the common bile duct and common hepatic artery were dissected. The gastroduodenal artery and the right gastric artery were transected. The pancreas was divided at the neck, while the pancreatic duct was identified for reconstruction. The jejunum was divided $10-15 \mathrm{~cm}$ distal to the Treitz ligament with an endoscopic linear stapler and the uncinate process was divided from superior mesenteric artery to complete the resection. For the reconstruction, the pancreaticojejunostomy was carried out using the duct-to-mucosa method (2). A laparoscopic choledochojejunostomy and gastrojejunostomy were performed with running suturing. During the surgery, $\sim 50 \mathrm{ml}$ of pale yellow ascites was observed. No invasion of the superior mesenteric vessels and no metastasis to the abdominal organs was detected. Only mild swelling in the head of pancreas was present. A tLPPPD R0 resection and lymphadenectomy were successfully achieved.

At post-operative day 1 (POD 1), the laboratory results showed a TB level of $53.5 \mu \mathrm{mol} / 1$ and a DBIL level of $50.2 \mu \mathrm{mol} / 1$. The drainage amylase test reached a peak level of $1583 \mathrm{IU} / 1$ (normal range, $\leq 3$ times level of serum amylase) with normal vital signs at POD 2. The TB level had decreased to a normal level of $27.5 \mu \mathrm{mol} / \mathrm{l}$ at POD 10 , with a low level of drainage amylase of $130 \mathrm{IU} / 1$ at POD 4. According to the Clavien grading system (7), no fatal complications, including heart failure, pulmonary infection, hemorrhage, pancreatic leakage and bile leakage, were observed, with the exception 
of grade I pancreatic leakage at POD 2. The post-operative pathological examination revealed a moderately-differentiated adenocarcinoma involving the pancreatic parenchyma (8), and negative findings in the lymph nodes. The tissues were sent to a pathologist at the West China Hospital, Sichuan University, for assessment by immunohistochemical staining. Immunohistochemical staining with hematoxylin and eosin revealed that the adenocarcinoma cells originated from pancreatic ductal epithelial cells, due to testing positive for the S100 protein and maspin and negative for CD10. The patient was uneventfully discharged at POD 12 with a diagnosis of pancreatic uncinate adenocarcinoma [T2N0M0, stage IB, according to the Clavien grading system (9)]. During 2 years of follow-up, abdominal enhanced magnetic resonance imaging and blood tumor markers revealed that the patient had no tumor recurrence or metastasis.

\section{Discussion}

Despite its complexity, tLPPPD has been reported to be a safe and feasible surgery following improvements in laparoscopic technology and greater surgical experience (5). Previous studies have shown a $1.6-8.0 \%$ rate of perioperative mortality $(10,11)$. While for low-grade malignant pancreatic head disease cases, the mortality rate for tLPPPD is comparable with the historical data for patients who underwent open pancreatoduodenectomy (5). The majority of studies with large series of patients with advanced periampullary malignant diseases present comparable findings in the oncological outcomes between tLPPPD and open pancreatodudenectomy (2). Palanivelu et al (10) reported a mean of 13 lymph nodes harvested and a 5-year survival rate of $32 \%$ for all patients. In addition, these authors noted a 5 -year survival rate of $31.7 \%$ for periampullary adenocarcinoma, $33.3 \%$ for pancreatic cystadenocarcinoma, $19.1 \%$ for pancreatic head adenocarcinoma and $50.0 \%$ for common bile duct adenocarcinoma. For 45 patients with advanced periampullary malignancies, Kendrick and Cusati (2) reported the retrieval of 15 median lymph nodes with an $89 \%$ margin-negative resection, a $16 \%$ rate for hepatic or pulmonary recurrence within a mean period of 7.4 months, and no port-site recurrence. Additional details concerning studies that have reported patients treated with tLPPPD are revealed in Table I.

In the past, an age of $>80$ years may have been considered as a contradiction for complex surgeries, such as pancreatoduodenonectomy, hepatectomy and organ transplantations. Elderly patients theoretically have a lower capability to tolerate complex procedures. The impairment of heart and pulmonary reserve function often leads to a high risk of post-operative cardiopulmonary complications. Additionally, with a soft pancreas and small pancreatic duct, this group of patients may experience pancreatic leakage, which is extremely detrimental. In the present case, due to an early diagnosis and mild jaundice, a soft pancreas and nearly normal pancreatic duct were detected during the surgery, and an end-to-side pancreaticojejunostomy was successfully performed. A slight increase in the drainage amylase level at POD 2 quickly decreased to a normal level at POD 4. This may have been a result of the surgeon's experience with the applied technology, which extended to $>50$ tLPPPD cases. The surgical duration was $6.5 \mathrm{~h}$ and no blood transfusion was necessary. From experience of another 4 successful cases of tLPPPD performed early last year in patients $>75$ years old (Table II), and despite the pre-operative impairment of cardiopulmonary function, we hypothesize that four potential factors may be responsible for the good outcome of the present 89-year-old patient: i) Experience in the relevant surgical skills; ii) a short surgical duration; iii) advanced post-operative cardiopulmonary management, and iv) similar to previous studies $(5,11)$, a moderately-differentiated adenocarcinoma that is not a contraindication for tLPPPD. In conclusion, tLPPPD is safe and feasible in selective elderly patients. Use of this technique may expand the number of patients who can undergo surgery and provide benefits to these patients.

\section{References}

1. Gagner M and Pomp A: Laparoscopic pylorus-preserving pancreatoduodenectomy. Surg Endosc 8: 408-410, 1994.

2. Kendrick ML and Cusati D: Total laparoscopic pancreaticoduodenectomy: Feasibility and outcome in an early experience. Arch Surg 145: 19-23, 2010.

3. Zureikat AH, Breaux JA, Steel JL and Hughes SJ: Can laparoscopic pancreaticoduodenectomy be safely implemented? J Gastrointest Surg 15: 1151-1157, 2011.

4. Asbun HJ and Stauffer JA: Laparoscopic vs. Open pancreaticoduodenectomy: Overall outcomes and severity of complications using the Accordion Severity Grading System. J Am Coll Surg 215: 810-819, 2012.

5. Kim SC, Song KB, Jung YS, Kim YH, Park do H, Lee SS, Seo DW, Lee SK, Kim MH, Park KM and Lee YJ: Short-term clinical outcomes for 100 consecutive cases of laparoscopic pylorus-preserving pancreatoduodenectomy: Improvement with surgical experience. Surg Endosc 27: 95-103, 2013.

6. Tee MC, Croome KP, Shubert CR, Farnell MB, Truty MJ, Que FG, Reid-Lombardo KM, Smoot RL, Nagorney DM and Kendrick ML: Laparoscopic pancreatoduodenectomy does not completely mitigate increased perioperative risks in elderly patients. HPB (Oxford) 17: 909-918, 2015.

7. Clavien PA, Camargo CA Jr, Croxford R, Langer B, Levy GA and Greig PD: Definition and classification of negative outcomes in solid organ transplantation. Application in liver transplantation. Ann Surg 220: 109-120, 1994.

8. Hruban RH, Adsay NV, Albores-Saavedra J, Compton C, Garrett ES, Goodman SN, Kern SE, Klimstra DS, Klöppel G, Longnecker DS, et al: Pancreatic intraepithelial neoplasia: A new nomenclature and classification system for pancreatic duct lesions. Am J Surg Pathol 25: 579-586, 2001.

9. Clavien PA, Camargo CA, Jr., Croxford R, Langer B, Levy GA and Greig PD: Definition and classification of negative outcomes in solid organ transplantation. Application in liver transplantation. Ann Surg 220: 109-120, 1994.

10. Palanivelu C, Jani K, Senthilnathan P, Parthasarathi R, Rajapandian S and Madhankumar MV: Laparoscopic pancreaticoduodenectomy: Technique and outcomes. J Am Coll Surg 205: 222-230, 2007.

11. Dulucq JL, Wintringer P and Mahajna A: Laparoscopic pancreaticoduodenectomy for benign and malignant diseases. Surg Endosc 20: 1045-1050, 2006. 